\title{
Electron Relaxation and Molecular Vibrations: Triatomic Systems of the First-Row Elements, the Methyl Radical, and Diazomethane and Ketene
}

\author{
JEREMY BuRdetT* \\ Department of Chemistry, University of Michigan, Ann Arbor, Michigan 48104
}

(Received 4 June 1969)

\begin{abstract}
The well-known second-order perturbation method is used to correlate the bond-bond interaction constants in ground and excited electronic states of triatomic systems of the first-row elements, and unusual force constants in diazomethane and ketene with the effect of electron relaxation. The third- and fourthorder perturbation schemes are used to determine qualitatively the contributions to the third-and fourthorder parts of the vibrational potential function of carbon dioxide, by the electron relaxation effect. The fourth-order theory is used to rationalize the negative anharmonicities observed in ketene, diazomethane, the methyl radical, and planar excited states of ammonia.
\end{abstract}

\section{INTRODUCTION}

Several polyatomic systems have been noted in the literature as exhibiting low and unusual force constants in some of their vibrational modes. Quite frequently, associated with such observations is the presence of abnormal anharmonicities with a corresponding poor fit to an uncorrected force field on isotopic substitution. In some systems negative anharmonicities have been observed, a phenomenon which is quite unusual.

The basis of our approach will be the pseudo-JahnTeller formalism, which predicts a softening of symmetry force constant when the electrons are allowed to relax, of the form

$$
\begin{aligned}
F_{i i}(k)=\left\langle k\left|\mathcal{F}_{i i}\right|\right. & k\rangle \\
& -2 \sum_{s}^{\prime}\left[\left|\left\langle k\left|\mathcal{H}_{i}\right| s\right\rangle\right|^{2} /\left(E_{s}^{(0)}-E_{k}^{(0)}\right)\right],
\end{aligned}
$$

for the $k$ th electronic state of a molecule. If we consider only one perturbing state $(n)$ and the ground state $(0)$, then in a two-state approximation, for the $i$ th symmetry coordinate $S_{i}$,

$F_{i i}(0)=\left\langle 0\left|\mathfrak{F}_{i i}\right| 0\right\rangle-2\left[\left|\left\langle 0\left|\mathfrak{H}_{i}\right| n\right\rangle\right|^{2} /\left(E_{n}{ }^{(0)}-E_{0}{ }^{(0)}\right)\right]$

and

$F_{i i}(n)=\left\langle n\left|\mathcal{F}_{i i}\right| n\right\rangle+2\left[\left|\left\langle 0\left|\mathcal{K}_{i}\right| n\right\rangle\right|^{2} /\left(E_{n}{ }^{(0)}-E_{0}^{(0)}\right)\right]$.

$\mathcal{F}_{i}$ and $\mathcal{F}_{i i}$ are the first and second derivatives of the electronic Hamiltonian with respect to $S_{i}$, taken at the equilibrium position $S_{i}=0$. The superscripts represent equilibrium values.

An analysis of triatomic systems to second order has been performed by Bader, ${ }^{1}$ using Eq. (1) to rationalize the sign of the bond-bond interaction constant, in several systems, and we shall use his approach to investigate the applications of Eq. (1) for the electronic ground state, and its natural extension, Eq. (2) for the upper excited state, for the systems $\mathrm{BO}_{2}$ and $\mathrm{CO}_{2}{ }^{+}$.

The fact that significant effects are observed in second order suggests that the formalism may be useful in the analysis of higher-order terms. The third-order contribution has been obtained by Salem ${ }^{2}$ via differentiation of Eq. (1). In this paper we use the higher-order perturbation treatment, and attempt to correlate the occurrence of unusual potential terms in higher order, with a significant second-order softening.

\section{FIRST-ROW TRIATOMIC SYSTEMS}

The molecules, radicals, and ions of this type may be listed according to the number of electrons in their valence shell, or more strictly according to their electronic structure in the ground state. The predicted and observed first excited states (those of a reasonably low $\Delta \epsilon)$ and a list of electronic configurations are given by Herzberg. ${ }^{3}$ It is noted that between 14 and 15 electrons a reversal of the order of the $1 \pi_{u}$ and $3 \sigma_{u}$ orbitals occurs.

For $D_{\infty h}$ systems the normal modes transform as $\Gamma_{\mathrm{vib}}=\Sigma_{0}{ }^{+}+\Sigma_{u}{ }^{+}+\Pi_{u}$. These species therefore are the permitted species of $\mathfrak{H}_{i}$. In order for any perturbations to be observed as a lowering of symmetry force constant, one or more of these representations must be contained in the product $\Gamma_{0}{ }^{*} \otimes \Gamma_{n}$ of the representations of the eigenfunctions corresponding to the state 0 and $n$ of the unperturbed system. In general, the electronic ground and excited states for these systems is dependent upon the number of valence-shell electrons, and thus the conclusions arrived at for one system should be applicable to isoelectronic molecules. Since a large number of the systems are radicals and unstable species, most of the vibrational data derive from studies of the electronic spectrum in the gas phase or infrared studies on matrix-isolated species.

The second-order perturbation term, as applied to 12-16-electron triatomics, predicts a $\sigma_{u}$ or $\sigma$ softening, manifest in lowering of $\nu_{3}$, the antisymmetric linear mode in the ground state of the molecule, for all the 
TABLE I. $\sigma_{u}$ softenings in linear systems.

\begin{tabular}{|c|c|c|c|c|c|}
\hline System & $\begin{array}{c}\nu_{1} \\
\left(\mathrm{~cm}^{-1}\right)\end{array}$ & $\begin{array}{c}\nu_{3} \\
\left(\mathrm{~cm}^{-1}\right)\end{array}$ & Ref. & Softening & $\begin{array}{c}\text { " } \int_{12} " \\
(\operatorname{mdyn} / \AA) b\end{array}$ \\
\hline $\mathrm{C}_{3}(X)$ & 1230 & 2040 & 8 & nonea & 0.392 \\
\hline $\mathrm{CNC}(X)$ & \multicolumn{3}{|c|}{ No data on $\nu_{1}$ or $\nu_{3}$ available } & none & \\
\hline $\operatorname{NCN}(X)$ & \multicolumn{3}{|c|}{$\begin{array}{l}F_{33} \text { observed characteristic of } \mathrm{CN} \\
\text { single bond }\end{array}$} & $\sigma_{u}$ & \\
\hline $\mathrm{BO}_{2}(X)$ & 1070 & 1322 & 5 & $\sigma_{u}$ & 1.42 \\
\hline $\mathrm{BO}_{2}(A)$ & 994 & 2357 & 5 & $\sigma_{u}$ & -0.895 \\
\hline $\mathrm{CO}_{2}^{+}(X)$ & 1280 & 1469 & 6 & $\sigma_{u}$ & 1.96 \\
\hline $\mathrm{CO}_{2}+(A)$ & 1131 & 2731 & 6 & $\sigma_{u}$ & -1.56 \\
\hline $\mathrm{N}_{3}$ & \multicolumn{3}{|c|}{ No data on $\nu_{1}$ or $\nu_{3}$ available } & $\sigma_{u}$ & \\
\hline $\mathrm{CO}_{2}(X)$ & 1388 & 2349 & 15 & $\sigma_{u}$ & 0.872 \\
\hline $\mathrm{BO}_{2}^{-}-(X)$ & $\cdots$ & 1948 & 7 & $\sigma_{u}$ & $\cdots$ \\
\hline $\operatorname{CCO}(X)$ & 1071 & 1978 & 10 & $\sigma$ & 2.37 \\
\hline $\operatorname{CNN}(X)$ & 1241 & 2847 & 12 & $\sigma$ & -3.9 \\
\hline $\operatorname{NCO}(X)$ & 1275 & 1922 & 11 & $\sigma$ & 2.56 \\
\hline
\end{tabular}

a But see text.

${ }^{b}$ In this paper force constants have been taken from the literature when available. Otherwise they have been calculated.

systems under consideration except those containing 12 or 13 valence electrons ( $\mathrm{C}_{3}$ and $\mathrm{CNC}$, for example) when we consider just the first excited states given by Herzberg. ${ }^{3}$

Similarly via Eq. (2), by an extension of Baders argument ${ }^{1}$ we should expect to observe a negative bond-bond interaction constant in the excited state $n$ which contributes to a positive bond-bond interaction constant in the ground electronic state.

Observation of an unusually low $\nu_{3}$ have been made for $\mathrm{NCN},{ }^{4} \mathrm{BO}_{2},{ }^{5} \mathrm{CO}_{2}+.{ }^{6}$ The force constant $F_{33}$ in NCN has been calculated from infrared data and shown to be more like the constant expected for a $\mathrm{CN}$ single bond, rather than the double-bond nature expected. ${ }^{4}$ For $\mathrm{BO}_{2}$ there is a striking difference between $\nu_{3}$ and the same mode in $\mathrm{BO}_{2}{ }^{-}$with one more electron. ${ }^{7}$ The antisymmetric stretching frequency occurs in the latter at between 1948 and $1995 \mathrm{~cm}^{-1}$, depending upon the cation. For the ground-state ${ }^{2} \Pi_{0}$ of $\mathrm{BO}_{2}$ the same mode occurs at $1322 \mathrm{~cm}^{-1}$, and in the upper perturbing state, ${ }^{2} \Pi_{u}$ at $2357 \mathrm{~cm}^{-1}$. For $\mathrm{CO}_{2}$ the ground-state antisymmetric stretching frequency occurs at $2349 \mathrm{~cm}^{-1}$. For $\mathrm{CO}_{2}{ }^{+}$in its ground state, ${ }^{2} \Pi_{g}, \nu_{3}$ is found at 1468 and $2731 \mathrm{~cm}^{-1}$, in the upper perturbing state ${ }^{2} \Pi_{u}$. [All the values of $\nu_{3}$ in $\mathrm{BO}_{2}$ and $\mathrm{CO}_{2}{ }^{+}$were taken as $\frac{1}{2}\left(2 \nu_{3}\right)$. $] \mathrm{A}$ summary of these observations is given in Table $\mathrm{I}$.

Vibrational data on both ground and excited states are unfortunately only available for $\mathrm{CO}_{2}{ }^{+}$and $\mathrm{BO}_{2}$. Johns ${ }^{6}$ has studied $\mathrm{CO}_{2}{ }^{+}$and finds an excited $\tilde{A}{ }^{2} \Pi_{u}$ state at $28500.6 \mathrm{~cm}^{-1}$ above the ground state $\widetilde{X}^{2} \Pi_{g}$ with a ${ }^{2} \Sigma_{u}+$ state at $34597 \mathrm{~cm}^{-1}$ above the ground state. Johns has also studied $\mathrm{BO}_{2}{ }^{5}$ and finds the $\tilde{A} / \tilde{X}$ separation to be $18291.5 \mathrm{~cm}^{-1}$ above the ground state.
All the excited states have been shown to have the same geometry as the ground state $\left(D_{\infty}\right)$.

We now take a look in a little more detail at the data in Table I. One feature is that the interaction constant is not of the same magnitude in the ground and excited states, as predicted by a broad application of the theory for the isoelectronic systems $\mathrm{CO}_{2}{ }^{+}$and $\mathrm{BO}_{2}$ via Eqs. (1) and (2). In both cases it is smaller in the excited state. The cause of this is possibly the two-state approximation. Higher states may soften the antisymmetric mode in the excited state, thus reducing the value of " $f_{12}$ " in the $\tilde{A}$ state. Because of the smaller energy separation between $\tilde{A}$ and other excited electronic states their effect will be more pronounced here than in the ground state. The effect may also be the result of a repulsive potential between the nonbonded end atoms of the chain leading to a slight nonequivalence in $\left\langle k\left|\mathfrak{H C}_{11}\right| k\right\rangle$ and $\left\langle k\left|\mathfrak{H C}_{33}\right| k\right\rangle$ and $\mathrm{a}$ positive contribution to the bond-bond interaction constant $f_{12}$ in both states. Since the electron relaxation interaction constant is of different sign in both states, the result will be to remove the equality in absolute magnitude between the two parameters. That is, if the two-state approximation holds, the nonbonded contribution to $f_{12}$ is $+0.20 \mathrm{mdyn} / \AA$ and $+0.26 \mathrm{mdyn} / \AA$ for $\mathrm{CO}_{2}{ }^{+}$and $\mathrm{BO}_{2}$, respectively, averaged over ground and excited states.

It may also be noted that a much smaller value of the interaction constant is observed ${ }^{8}$ in the radical $C_{3}$ where no interaction with first excited states can occur by symmetry. This is positive and thus could arise from a higher-energy ${ }^{1} \Sigma_{u}{ }^{+}$state of the system than we have considered. Such a state would be contained in 
the configuration $\left(4 \sigma_{g}\right)^{2}\left(1 \pi_{u}\right)^{3}\left(3 \sigma_{u}\right)^{2}\left(1 \pi_{g}\right)$. In view of the probable large energy scparation, such electronic: softening should be small by a consideration of Baders arguments. ${ }^{9}$ No data are available on this unobserved excited state, so it is impossible to say whether this interaction constant is due to the electronic relaxation or to nonbonded effects. If due to nonbonded effects, it is roughly twice the size contribution $(\sim 0.4 \mathrm{mdyn} / \AA)$ as needed to explain the interaction constant difference in ground and excited states for $\mathrm{BO}_{2}$ and $\mathrm{CO}_{2}{ }^{+} .(0.26$ and $0.2 \mathrm{mdyn} / \AA$, respectively).

For the 14-electron systems $\mathrm{CCO}^{10}$ and the 15electron system $\mathrm{NCO},{ }^{11}$ agreement with Bader's qualitative scheme is complete. However, an extremely unusual feature is furnished by the 14-electron radical $\mathrm{CNN}$ which has a large negative value of the bondbond interaction constant. The results are from Jacox and Milligan's matrix-isolation work on the radical,12 and the result of the negative interaction constant is the high antisymmetric stretching frequency of the radical. These authors compare its value to the antisymmetric stretching modes in excited states of $\mathrm{BO}_{2}$ and $\mathrm{CO}_{2}{ }^{+}$, which as we have seen are in the direction expected of the relaxation effect in an upper perturbing state for this series of systems. However, the frequency reported for $\mathrm{CNN}$ is presumably for the ground state since the radical is trapped in a solid matrix, and is certainly in the opposite direction to what would be expected, unless the radical is in a metastable excited state. This result for CNN is certainly anomalous.

For $\mathrm{OF}_{2}$ and ${ }^{13} \mathrm{CF}_{2}$ the large bond-bond interaction constant is responsible for the unusual observation that $\nu_{1}>\nu_{3}$. For $\mathrm{CCl}_{2}{ }^{13}$ and $\mathrm{OCl}_{2}$, however, if the frequencies are as assigned, $\nu_{3}>\nu_{1}$.

\section{THE POTENTIAL FUNCTION OF CARBON DIOXIDE}

The third-order contribution to the potential function by the electronic relaxation may be determined by differentiation of the pseudo-Jahn-Teller equation. ${ }^{2}$ However, we shall find it convenient to use the thirdand fourth-order perturbation expressions in discussing higher potential terms.

By a procedure exactly analagous to the derivation of the second-order pseudo-Jahn-Teller expression we find that the contribution to terms of order $S_{i}{ }^{3}$ is

$$
S_{i}{ }^{3}\left(\frac{1}{6}\left\langle k\left|\mathfrak{K}_{i i i}\right| k\right\rangle-\sum_{s} \frac{\left\langle k\left|\mathfrak{F}_{i i}\right| s\right\rangle\left\langle s\left|\mathfrak{F}_{i}\right| k\right\rangle}{E_{s}{ }^{(0)}-E_{k}{ }^{(0)}}+\sum_{s}^{\prime} \sum_{i}^{\prime} \frac{\left\langle k\left|\mathfrak{F}_{i}\right| s\right\rangle\left\langle s\left|\mathfrak{K}_{i}\right| t\right\rangle\left\langle t\left|\mathfrak{K}_{i}\right| k\right\rangle}{\left(E_{s}{ }^{(0)}-E_{k}{ }^{(0)}\right)\left(E_{t}{ }^{(0)}-E_{k}{ }^{(0)}\right)}\right)
$$

by third-order perturbation theory. In this equation we have made use of the fact that the first-order energy correction appearing in the general third-order perturbation energy correction is zero, since no true (first-order) Jahn-Teller effect exists in the systems we shall consider. $\frac{1}{6}\left\langle k\left|\mathfrak{K}_{i i i}\right| k\right\rangle$ is of course the "classical" third-order contribution.

By a similar procedure the fourth-order contribution in $S_{i}$ is given by

$$
\begin{aligned}
& S_{i}{ }^{4}\left(\frac{1}{24}\left\langle k\left|\mathcal{K}_{i i i i}\right| k\right\rangle-\frac{1}{4} \sum_{s} \frac{\left|\left\langle k\left|\mathcal{K}_{i i}\right| s\right\rangle\right|^{2}}{E_{s}{ }^{(0)}-E_{k}{ }^{(0)}}-\frac{1}{3} \sum_{s}{ }_{s} \frac{\left\langle k\left|\mathcal{F}_{i}\right| s\right\rangle\left\langle s\left|\mathcal{K}_{i i i}\right| k\right\rangle}{E_{s}{ }^{(0)}-E_{k}{ }^{(0)}}\right.
\end{aligned}
$$

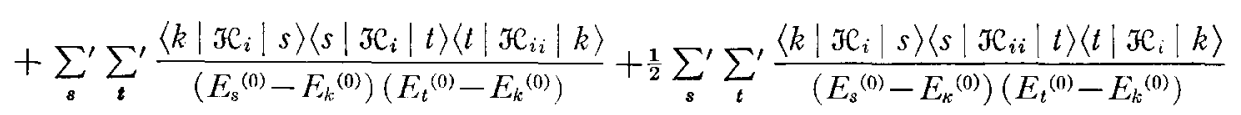

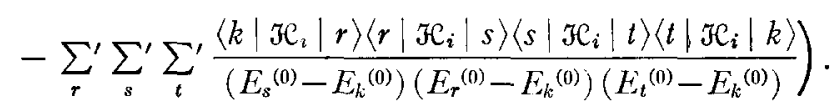

In this section we shall compare the electron realxation contributions to the magnitude of the various terms occurring in third and fourth order in the potential function of carbon dioxide analyzed empirically by Dennison. ${ }^{15}$ Since pseudo-Jahn-Teller interaction is only predicted for the antisymmetric linear mode, from previous arguments the matrix elements $\left\langle k\left|\mathfrak{K}_{i}\right| s\right\rangle$ will only be significant if allowed to be nonzero on symmetry grounds and if $\mathfrak{K}_{2}=\mathfrak{J C}_{3}$. We can use the equations developed above to calculate these contributions and will arrive at a series of matrix element products. It is not the purpose of this paper to evaluate these numerically, and we shall parametrize the data to obtain some accessible results. As an approximation, which we trust will be valid, we shall put all the pseudoJahn-Teller softening terms $\left\langle X\left|\mathscr{F}_{3}\right|^{1} \Sigma_{u}{ }^{+}\right\rangle$equal to $\alpha$; the diagonal force constants $\left\langle k\left|\mathfrak{H}_{33}\right| k\right\rangle$, and also the transition diagonal force constants $\left\langle k\left|\mathfrak{F}_{33}\right| m\right\rangle$, equal to $\beta$. We shall also use an average value of the energy separation $E_{m}^{(0)}-E_{k}^{(0)}$ of $\epsilon$ when $k$ is the ground state $(\epsilon>0)$.

Using Dennison's nomenclature the third- and fourthorder parts of the vibrational potential function are

$$
\begin{gathered}
a \sigma^{3}+b \rho^{2} \sigma+c \sigma \zeta^{2}, \\
d \sigma^{4}+e \rho^{4}+f \zeta^{4}+g \sigma^{2} \rho^{2}+h \sigma^{2} \zeta^{2}+i \rho^{2} \zeta^{2},
\end{gathered}
$$


respectively, where $\sigma=S_{1}, \zeta=S_{3}, \rho^{2}=\left[S_{2 a}{ }^{2}+S_{2 b}{ }^{2}\right]\left(S_{2 a}\right.$ and $S_{2 b}$ are the two orthogonal bending modes of the molecule).

By the analysis of a large number of infrared absorption bands, the following values of these coefficients have been obtained: $a=-3.0 ; b=71.3 ; c=-250 ; d=$ $1.5 ; e=0.5 ;-3.7$ (from two different methods of calculation) $f=6.4 ; g=1.9 ; h=8.9$; and $i=-25.7$.

To simplify discussion we shall limit ourselves to a basis set containing the three predicted first excited states of $\mathrm{CO}_{2}$. From Ref. 3 these can be seen to be ${ }^{1} \Delta_{u}$, ${ }^{1} \Sigma_{u}{ }^{-},{ }^{1} \Sigma_{u}{ }^{+}$, and ${ }^{1} \Pi_{g}$, with a ground state of ${ }^{1} \Sigma_{g}{ }^{+}$. Since no excited states of species ${ }^{1} \Sigma_{g}+$ are contained in the basis set the electronic contribution to " $a$ " will be zero, from Eq. (3.)

Similarly the absence of an excited ${ }^{1} \Pi_{u}$ state implies a zero contribution to " $b$." The presence of an excited ${ }^{1} \Sigma_{u}{ }^{+}$state, however, means that the electronic contribution to " $c$ " will certainly not be zero. This contribution can be seen to be

$$
-\left\langle X\left|\mathcal{F}_{13}\right|{ }^{1} \Sigma_{u}{ }^{+}\right\rangle\left\langle{ }^{1} \Sigma_{u}{ }^{+}\left|\mathcal{F}_{3}\right| X\right\rangle /\left(E^{1} \Sigma_{\Sigma^{+}}{ }^{(0)}-E_{X^{(0)}}\right),
$$

where $\widetilde{X}$ is the ${ }^{1} \Sigma_{g}{ }^{+}$ground state. This is negative and proportional to the second-order softening term. We therefore expect this contribution to the third-order terms to be large since the $\sigma_{u}$ softening of $\mathrm{CO}_{2}$ in its ground state is very noticeable. These results are indeed reproduced in Dennison's values of " $a$, , " $b$," and "c." The value of " $c$ " is an order of magnitude larger than " $b$ " and two orders of magnitude larger than " $a$." The most important feature is that " $c$ " is large and negative; because of the absence of excited ${ }^{1} \mathrm{II}_{u}$ and ${ }^{1} \Sigma_{g}+$ states, the electron relaxation contributions to " $d$, , " $e$ " and " $g$ " are zero and it is interesting to see that from Dennison's data " $d$, , " $e$," and " $g$ " have the lowest values of all the fourth-order coefficients. The electronic contribution to " $f$ " is however nonzero and is, using the same parameters as before,

$$
\frac{1}{3}\left(\alpha^{2} / \epsilon\right)+\frac{1}{2}\left(\alpha^{2} \beta / \epsilon^{2}\right)=\left(\alpha^{2} / \epsilon\right)\left[(2 \epsilon)^{-1} \beta-\frac{1}{3}\right] .
$$

Now $\beta$ in this case represents the matrix element $\left\langle{ }^{1} \Sigma_{u}{ }^{+}\left|{ } \mathcal{F}_{33}\right|{ }^{1} \Sigma_{u}{ }^{+}\right\rangle$which is the classical force constant for the antisymmetric mode. This is about $10 \mathrm{mdyn} / \AA$ or $10^{-10} \mathrm{erg} / \AA^{2}$. Putting the ${ }^{1} \Sigma_{u}{ }^{+}$state at an energy separation above the ground state equal to the ionization potential, a calculation arrives at the conclusion that the quantity in brackets is positive and hence that the contribution to " $f$ " is positive. For an energy separation lower than the ionization potential (i.e., all sensible estimates) the contribution is correspondingly larger. From Dennison's work we see that the value of " $f$ " is positive and larger than " $d, "$ " $e$, , and " $g . "$ Now by expanding the Hamiltonian in terms of $S_{i}$ and $S_{j}$ we may evaluate the fourth-order contribution to the potential expansion by terms $S_{i}{ }^{2} S_{j}{ }^{2}$. Applying the usual restrictions on the various states $r, s, t$ we find that the electronic contribution to " $h$ " is also nonzero and is the sum of three terms

$$
\begin{aligned}
& -2 \frac{\left|\left\langle X\left|\mathcal{F}_{13}\right|{ }^{1} \Sigma_{u}{ }^{+}\right\rangle\right|^{2}}{E^{1} \Sigma_{u}{ }^{(0)}-E_{X}{ }^{(0)}} \\
& -\frac{\left\langle X\left|\mathcal{F C}_{3}\right|{ }^{1} \Sigma_{u}+\right\rangle\left\langle{ }^{1} \Sigma_{u}{ }^{+}\left|\mathcal{F C}_{113}\right| X\right\rangle}{E^{1} \Sigma_{u}{ }^{(0)}-E_{X}{ }^{(0)}} \\
& \quad+\frac{3\left\langle X\left|\mathcal{F C}_{3}\right|{ }^{1} \Sigma_{u}{ }^{+}\right\rangle \mid{ }^{2}\left\langle\Sigma_{u}{ }^{+}\left|\mathcal{F C}_{11}\right|{ }^{1} \Sigma_{u}{ }^{+}\right\rangle}{\left(E^{1} \Sigma_{u}{ }^{+(0)}-E_{X}{ }^{(0)}\right)^{2}} .
\end{aligned}
$$

Now the first term represents a transition interaction constant. Since the diagonal interaction constants in these systems are small, we should expect this term to be small also. As an overestimate of its value we shall assign it the value of $\beta / 10$, being the approximate magnitude of the total interaction constant in $\mathrm{CO}_{2}$. We can then see that its contribution to " $h$ " will be negligible, compared to the other two terms. Using the same method as used above for " $f$," the sum of the second and third terms becomes

$$
\frac{3}{2}\left(\alpha^{2} / \epsilon\right)\left[(\beta / \epsilon)-\frac{2}{3}\right],
$$

which is positive for any sensible estimate of the energy separation of the ${ }^{1} \Sigma_{u}-$ state from the ground state. For any value of $\epsilon$ less than the ionization potential of around $150000 \mathrm{~cm}^{-1} 16$ the electronic contribution to " $h$ " is larger than to " $f$ " by consideration of the quantities in brackets. It is pleasing to note that both " $f$ " and " $h$ " are positive in Dennison's computations and that " $f$ " is indeed smaller than " $h$ " although the difference is not very large.

Similarly, the electronic contribution to " $i$ " is nonzero and is also the sum of three terms,

$$
\begin{aligned}
& +\frac{3}{2} \frac{\left|\left\langle X\left|\mathcal{F}_{3}\right|{ }^{1} \Sigma_{u}{ }^{+}\right\rangle\right|{ }^{2}\left\langle{ }^{1} \Sigma_{u}\left|\mathcal{F C}_{22}\right|{ }^{1} \Sigma_{u}{ }^{+}\right\rangle}{\left(E^{1} \Sigma_{\Sigma_{u}}{ }^{(0)}-E_{X}{ }^{(0)}\right)^{2}} \\
& -\frac{\left\langle X\left|\mathcal{F}_{3}\right|{ }^{1} \Sigma_{u}{ }^{+}\right\rangle\left\langle{ }^{1} \Sigma_{u}+\left|\mathcal{F}_{322}\right| X\right\rangle}{E^{1} \Sigma_{u}{ }^{+(0)}-E_{X}{ }^{(0)}} \\
& -6 \frac{\left|\left\langle X\left|\mathcal{F C}_{3}\right|^{1} \Sigma_{u}{ }^{+}\right\rangle\right|^{2}\left|\left\langle{ }^{1} \Sigma_{u}{ }^{+}\left|\mathcal{F C}_{22}\right|^{1} \mathrm{II}_{0}\right\rangle\right|^{2}}{\left(E^{1} \Sigma_{u}{ }^{(0)}-E_{X}{ }^{(0)}\right)^{2}\left(E^{1} \Pi_{g}{ }^{(0)}-E_{X}{ }^{(0)}\right)} .
\end{aligned}
$$

If we now write $\beta_{\pi}$ as the bending force constant and $\epsilon_{\boldsymbol{*}}$ as the energy separation of the ${ }^{1} \Pi_{g}$ state from the ground state the contribution can be written

$$
\frac{3}{2}\left(\alpha^{2} \beta_{\pi} / \epsilon^{2}\right)\left[1-\left(4 \beta_{\pi} / \epsilon_{\pi}\right)\right]-\left(\alpha^{2} / \epsilon\right) .
$$

The bending force constant in this molecule is about $0.65 \times 10^{-11} \mathrm{erg} / \mathrm{rad}^{2}$, and so we find the term in brackets to be positive for all values of $\epsilon$ less than 130000 $\mathrm{cm}^{-1}$. It is unlikely that the energy separation is that high and so we may conclude that this first term and hence the entire electron relaxation contribution to $i$ will be negative. Dennison finds a large negative value of " $i$ " in his potential analysis. ${ }^{15}$ 
We have therefore shown that the signs and in some cases the relative magnitude of the electron relaxation contributions to the vibrational potential terms of third and fourth order in the $\mathrm{CO}_{2}$ molecule fit extremely well with the empirical calculations of Dennison for the total anharmonic constants up to fourth order. We cannot say how large a part these electronic effects play in determining the structure of the potential function at this qualitative stage in the development of the theory. However the excellent correlation obtained in this section suggests that where electronic relaxation effects are important in the second-order term, the signs and magnitudes of the other potential coefficients may well be primarily determined by the electronic relaxation terms, in such triatomic species. (The effect may be even more apparent in $\mathrm{BO}_{2}$ and $\mathrm{CO}_{2}{ }^{+}$where the second-order term seems considerably larger.)

\section{THE METHYL RADICAL}

The methyl radical has been prepared in an argon matrix by Jacox and Milligan ${ }^{16}$ by the vacuum-ultraviolet photolysis of methane and by Andrews and Pimental as a radical complex associated with alkali halide molecules. ${ }^{17}$

The former authors calculated a ground-state force constant of $0.177 \mathrm{mdyn} / \AA$ for the out-of-plane deformation ( $\left.a_{2}^{\prime \prime}\right)$ mode of $\mathrm{CH}_{3}$ corresponding to a frequency of $611.0 \mathrm{~cm}^{-1}$. This was the only fundamental observed in their study, and the force constant was calculated assuming a planar $D_{3 h}$ geometry as suggested by the ultraviolet absorption work of Herzberg ${ }^{18}$ and the electron spin resonance studies of Fessenden and Schuler. ${ }^{19}$ These conclude that the radical was either planar or very nearly planar.

However, on comparison with the observed frequencies of ${ }^{12} \mathrm{CH}_{3},{ }^{13} \mathrm{CD}_{3},{ }^{12} \mathrm{CD}_{3},{ }^{12} \mathrm{CHD}_{2}$, and ${ }^{12} \mathrm{CH}_{2} \mathrm{D}$, the planar model did not look so convincing due to the discrepancies which were quite large between the calculated and observed frequencies. A pyramidal model showed better agreement with observation, but a nonplanarity greater than that in ground-state ammonia was the result. This did not fit into the results of Herzberg and of Fessenden and Schuler, and was extremely unlikely on the basis of Walsh's scheme. ${ }^{20}$ This deviation, if explained on the grounds of anharmonicity, was in the opposite direction to that usually observed, and Jacox and Milligan were forced to conclude that only positive even powers contributed to the potential function of $\mathrm{CH}_{3}$. Precedents for this explanation lay in the studies of Walsh and Warsop on excited planar states of ammonia, ${ }^{21}$ and in Moore and Pimentel's analysis of diazomethan ${ }^{22}$ and ketene. ${ }^{23}$

Pseudo-Jahn-Teller interaction should occur in planar $\mathrm{CH}_{3}$ from a consideration of the electronic states. The ground state of the radical is ${ }^{2} A_{2}{ }^{24}$ as one would expect from consideration of electronic configuration.
Three excited ${ }^{1} A_{1}{ }^{\prime}$ states have also been identified, the lowest with an energy separation above the ground state of $46205 \mathrm{~cm}^{-1}$. The effect should be smaller than for ammonia, where the second-order term in Eq. (1) is so large that the planar ground-state geometry is unstable and a pyramidal species is observed. The transition density is smaller by an approximate factor of $2^{-1 / 2}$ for methyl (as is simply shown by expressing the perturbation term on a molecular-orbital basis similar to Baders expression for $\mathrm{CO}_{2}{ }^{2}$ ).

The negative anharmonicity is neatly handled by the present approach. Matrix elements of the form $\left\langle s\left|\mathcal{F C}_{2}\right| t\right\rangle$ are nonzero provided $\langle s|$ transforms as $a_{2}^{\prime \prime}$ and $|t\rangle$ as $a_{1}^{\prime}$. Matrix elements containing any other $\mathcal{F}_{i}$ will be small or zero since the ground-state contribution to the transition density lies solely in a $p_{2}$ carbon orbital, which lies out of the plane of the $\mathrm{CH}_{3}$ system. The terms in $S_{2}{ }^{3}$ will be identically zero by symmetry. Initially we examine terms in $S_{2}{ }^{4}$.

As a basis set of eigenfunctions to use in the perturbation expansion we will consider an ${ }^{2} A_{2}{ }^{\prime \prime}$ ground state and the two excited states arising from promotion of the $a_{2}{ }^{\prime \prime}$ electron to the antibonding $\bar{a}_{1}^{\prime}$ orbital $\left(\widetilde{B}^{2} A_{1}{ }^{\prime}\right)$ and antibonding $\bar{e}^{\prime}$ orbital $\left(\tilde{C}^{2} E^{\prime}\right)$.

The force constant for the out-of-plane bending mode $a_{2}^{\prime \prime}$ is

$$
\begin{aligned}
F_{2 E}(0)=\left\langle X\left|\mathcal{F}_{22}\right|\right. & X\rangle \\
& -2\left[\left|\left\langle X\left|\mathcal{F}_{2}\right| B\right\rangle\right|^{2} /\left(E_{B}{ }^{(0)}-E_{X}{ }^{(0)}\right)\right] .
\end{aligned}
$$

The fourth-order contribution to $S_{i}^{4}$ then becomes, using Eq. (4),

$$
\begin{aligned}
& \frac{1}{24}\left\langle X\left|\mathcal{H C}_{2222}\right| X\right\rangle \\
& \quad-\frac{1}{3}\left[\left\langle X\left|\mathfrak{H C}_{2}\right| B\right\rangle\left\langle B\left|\mathfrak{F C}_{222}\right| X\right\rangle /\left(E_{B}{ }^{(0)}-E_{X^{(0)}}\right)\right] \\
& \quad+\frac{1}{2}\left[\left|\left\langle X\left|\mathfrak{H C}_{2}\right| B\right\rangle\right|^{2}\left\langle B\left|\mathfrak{H C}_{22}\right| B\right\rangle /\left(E_{B}{ }^{(0)}-E_{X}{ }^{(0)}\right)^{2}\right] .
\end{aligned}
$$

The first term is expected to be negative, and, on its own, is responsible for the normal positive anharmonicities observed in modes of this sort.

The second term is likely to be small since it contains a transition constant of third order, but the fourth term contains the second-order softening term multiplied by a diagonal force constant. It is expected to be large and positive.

Hence if the second-order softening parameter is large this term may be expected to dominate the above expression. This would lead to a positive over-all contribution to the potential function in fourth order and accounts for the observation of negative anharmonicity in this mode. Additional support for this view lies with the methyl alkali halide radicals $\mathrm{CH}_{3} \cdots \mathrm{MX}$ where unusual vibrational data are also observed. ${ }^{25}$

Using the fact that matrix elements $\left\langle X\left|\mathcal{T C}_{\imath}\right| n\right\rangle$ will only be significant for $\mathfrak{H}_{i}=\mathfrak{H C}_{2}$, the only nonzero contribution to the cross terms $S_{2}{ }^{2} S_{i}{ }^{2}$ (the only ones al- 


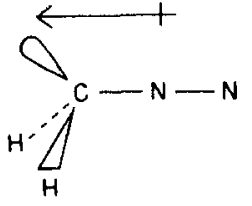

FIG. 1. The axial dipole in the $\mathrm{CH}_{2} \mathrm{~N}_{2}$ hydrogen wagging mode.

lowed to occur by symmetry) is from the expression

$$
\left|\left\langle X\left|\mathcal{F}_{2}\right| B\right\rangle\right|^{2}\left\langle B\left|\mathfrak{F}_{i i}\right| B\right\rangle /\left(E_{B}{ }^{(0)}-E_{X}(0)\right)^{2}
$$

which will be positive.

We have therefore successfully applied the theory to the methyl radical and showed, after simplification, that a positive contribution to the fourth-order terms in the potential expansion occurs when the effects of electronic relaxation are included and is dependent upon the size of the second-order softening.

A similar treatment to the above is directly applicable to the first ${ }^{1} A_{2}{ }^{\prime \prime}$ state of planar ammonia, where negative anharmonicities have been observed..$^{21}$ Groundstate ammonia exhibits no unusual effect since the $a_{2}{ }^{\prime \prime} / \bar{a}_{1}{ }^{\prime}$ separation (using $D_{3 h}$ labels) increases rapidly as the angle $\mathrm{HNH}$ drops from $120^{\circ}$. We should only expect to see significant effects where the system is planar or close to planar, due to this condition.

\section{DIAZOMETHANE AND KETENE}

The systems to be discussed in this section are diazomethane and ketene, which are very interesting from the viewpoint of their vibrational potential function. Initially problems in vibrational assignment were due to one of the hydrogen motions being at an unusually low frequency. Problems have also been met concerning the sign of some of the force constants, and noncompliance with product and sum rules.

$\mathrm{CH}_{2} \mathrm{~N}_{2}$ and $\mathrm{CH}_{2} \mathrm{CO}$ belong to the point group $C_{2 v}$ and hence the normal modes transform as $\Gamma_{\mathrm{vib}}=4 A_{1}+$ $3 B_{1}+2 B_{2}{ }^{26}$ The $B_{2}$ modes are the out-of-plane skeletal bend $\left(\nu_{8}\right)$ and the wagging vibration of the two hydrogen atoms $\left(\nu_{9}\right)$. The $B_{1}$ block contains the in-plane skeletal bend $\left(\nu_{7}\right)$, the $\mathrm{CH}_{2}$ rocking motion $\left(\nu_{6}\right)$, and the antisymmetric $\mathrm{CH}_{2}$ stretching mode $\left(\nu_{5}\right)$.

These systems are isolectronic with $\mathrm{CO}_{2}$ and by analogy to this the ground state of the molecule can be written

$$
\begin{aligned}
K K K\left(1 a_{1}\right)^{2}\left(1 b_{1}\right)^{2}\left(2 a_{1}\right)^{2}\left(3 a_{1}\right)^{2} & \\
& \times\left(2 b_{1}\right)^{2}\left(1 b_{2}\right)^{2}\left(3 b_{1}\right)^{2}\left(2 b_{2}\right)^{2} A_{1} .
\end{aligned}
$$

The lowest energy transitions in the ultraviolet spectrum will then be to ${ }^{1} A_{1}$ and ${ }^{1} A_{2}$ states by promotion of a $3 b_{1}$ or $2 b_{2}$ electron to the higher-energy $3 b_{2}$ and $4 b_{1}$ orbitals. 'Two higher states of species ${ }^{1} B_{2}$ may arise from promotion of $2 b_{2}$ electron to the orbitals $3 s a_{1}$ and $3 p a_{1}$. Two states of species ${ }^{1} B_{1}$ may similarly arise if the electron is from the $3 b_{1}$ orbital.

The electronic spectrum of both diazomethane and ketene, although themselves quite similar, have little resemblance to that of $\mathrm{CO}_{2}$. Merer ${ }^{27}$ has identified transitions of the type ${ }^{1} A_{1} \leftarrow^{1} A_{1},{ }^{1} B_{2} \leftarrow{ }^{1} A_{1}$, and ${ }^{1} B_{1} \longleftarrow^{1} A_{1}$ for diazomethane. For ketene all the observed and assigned transitions are parallel transitions, the perpendicular bands forming an unresolved background..$^{28}$ A detailed list of all the low-lying energy levels of these two molecules is unfortunately not available.

First of all we shall consider the two modes contained in the $B_{2}$ symmetry block, $\nu_{8}$ and $\nu_{9}$. Suitable electronic states exist, or at least are predicted to exist, to soften the ground-state force constants in these modes. Moore and Pimentel have analyzed the vibrational spectra of both ground-state ketene ${ }^{23}$ and ground-state diazomethane ${ }^{22}$ in detail and find that the $\mathrm{CH}_{2}$ wagging mode is indeed at an extremely low frequency for this sort of vibration. $\nu_{8}$, the out-of-plane skeletal bending motion, however, has a similar value of force constant in diazomethane, ketene, $\mathrm{CO}_{2}, \mathrm{~N}_{3}^{-}$, etc.

These authors found that $F_{99}$ the symmetry force constant was less than one-fifth the value found in ethylene (a prototype molecule they considered) for diazomethane, and less than one-half the ethylene value for ketene.

We can now consider the form of the perturbation in terms of the location of the transition density. In this case the $B_{2}$ density derived from interaction with the lowest ${ }^{1} B_{2}$ state is located on the $\mathrm{XYZ}$ skeleton, above and below the $\mathrm{H}_{2} \mathrm{Z}$ plane, in analogy with triatomic systems. On bending the $\mathrm{CH}_{2}$ group in this plane (as in the wagging mode), the configuration around the carbon atom will change, and if the amplitude of vibration is large enough the configuration will approach tetrahedral. The structure now contains a large dipole contribution from $I$ along with an enhanced dipole of species $B_{2}$ (Fig. 1). This axial dipole is large being the result of a large-scale charge separation, and a priori we should expect the second-order perturbation term to be large. This is indeed the case. In contrast, the out-of-plane skeletal NNC bending motion produces no exceptionally large dipole on exciting the vibration. The pseudo-Jahn-Teller effect on this basis is certainly expected to be much smaller than for the wagging mode.

The perturbing effect on $\nu_{9}$ is observed to be more pronounced in diazomethane than in ketene. The ion. ization potential is lower in the former than in ketene, resulting in a red shift of the ultraviolet bands of ketene with respect to those in diazomethane. The value of $\Delta \epsilon$ should then be smaller in $\mathrm{CH}_{2} \mathrm{~N}_{2}$ than in $\mathrm{CH}_{2} \mathrm{CO}$, and hence a larger $P_{99}(n)$ in $\mathrm{CH}_{2} \mathrm{~N}_{2}$ than in

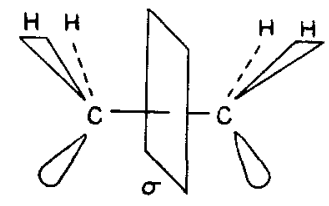

FIG. 2. Symmetry plane in the ethylene $b_{1 u}$ hydrogen mode. 
CH.CO. This is assuming that the magnitudes of the matrix elenents for the two cases are similar.

It is interesting at this stage to take a look at the ethylene system to see how valid the "normality" of the rocking and wagging modes is in this system. In brief, ethylene is predicted to possess excited states ${ }^{29}$ capable of softening both the wagging and rocking modes by a vibrational perturbation as discussed in this paper. Also the effect of electron rearrangement may be expected to be large when the hybridization changes concerned are compared to the case of diazomethane and ketene.

However, in contrast to the latter two molecules, the axial dipole produced in these modes in ethylene is identically zero from the symmetry of the system.

The transition density is located along the $\mathrm{C}-\mathrm{C}$ axis, in a similar fashion to diazomethane and ketene. Since the axial dipole is by symmetry zero (Figs. 2 and 3 ), no perturbation of the rocking and wagging force constants is expected on this basis.

So the use of $\mathrm{C}_{2} \mathrm{H}_{4}$ as a prototype may well be a good choice.

A similar analysis to the $B_{2}$ case holds for the $B_{1}$ block containing the in-plane skeletal bend, the $\mathrm{CH}_{2}$ rocking motion, and the antisymmetric $\mathrm{CH}_{2}$ stretching mode. The first two frequencies occur at much lower energy than does the hydrogen stretching motion, and can be validly factored out from $\nu_{5}$. Now the analysis is very similar to that for the two modes of the $B_{2}$ block. The perturbing charge field now lies in the plane of the $\mathrm{CH}_{2}$ group. The rocking motion of the latter produces a similar dipole to the wagging case (Fig. 4). The in-plane bend, like the out-of-plane analog, produces only a small dipole, relatively speaking. The force constant in this mode is of similar magnitude to the bending force constant in $\mathrm{CO}_{2}, \mathrm{~N}_{2} \mathrm{O}$, etc., and slightly smaller. The rocking mode in ketene, on the other hand, has a force constant roughly one-half the ethylene value, and a higher value in diazomethane. The magnitude of the effect in these two systems therefore seems to have been reversed, the ketene force constant being smaller than the corresponding figure for diazomethane. This is inexplicable at the present time, but may be resolved when the nature of the ${ }^{1} B_{1}$ perturbing state is examined in more detail. Merer, for example, tentatively suggested that the state he observed for diazomethane, and labeled ${ }^{1} B_{1}$, was in fact due to a two-electron transition. ${ }^{27}$

The intensity of $\nu_{9}$ and $\nu_{6}$ is one striking feature of the infrared spectrum of these molecules and is typical of wagging modes in this type of system where a large

FIG. 3. Inversion symmetry in the ethylene $b_{2 g}$ hydrogen mode.
Fic. 4. The axial dipole in the $\mathrm{CH}_{2} \mathrm{~N}_{2}$ hydrogen rocking mode.

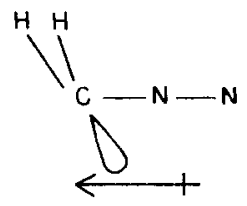

hybridization change occurs on vibration. However the high intensity of the overtones is unusual, in one case for deuterated diazomethane this is obviously apparent. ${ }^{30}$ Similar observations are reported for the system HD where the mathematical derivation of a nonzero dipole moment takes into account the electronic motion. . $^{31}$

The relaxation effect in the stretching modes is small. The transition required is a $\sigma$-type transition involving the $\mathrm{C}-\mathrm{H}$ bonds, and will be of higher energy than the $\pi$-type transitions occurring within the chain. As with the methyl stretching modes, the effect should be small.

A rationalization of the modes contained in the $A_{1}$ block is very much along the same lines as that for softenings in triatomic chains. In both ketene and diazomethane the interaction constant between the two linear skeletal modes is positive and of similar magnitude to the constants in the isoelectronic systems $\mathrm{N}_{3}-$ and $\mathrm{CO}_{2}$. The $\mathrm{NN}$ force constant is similar to that in $\mathrm{N}_{2} \mathrm{O}$ and that for the $\mathrm{CN}$ linkage is intermediate between single- and double-bond values. Decius ${ }^{24}$ has produced a correlation between bond length and force constant for NN bonds. Applying the same treatment to the $\mathrm{NN}$ bond in $\mathrm{CH}_{2} \mathrm{~N}_{2}$, the calculated $f_{\mathrm{NN}}$ is 17.65 mdyn $/ \AA$, to be compared to the observed value of $16.89 \mathrm{mdyn} / \AA$. This discrepancy of $6 \%$ is larger than the average discrepancy noted by Decius in the systems he considered, excluding those for which a pseudoJahn-Teller interaction is predicted. For $\mathrm{N}_{2} \mathrm{O}$ and $\mathrm{HN}_{3}$, discrepancies of $5 \%$ are observed, all in the same direction. This is evidence, perhaps slender, that the correlation between the static bond length and the dynamic force constant is not quite so simple when electronic effects are considered.

A brief summary of the vibrational potential function of these two molecules ${ }^{22,23}$ is pertinent.

$F_{99}$ has a value of $0.045 \times 10^{-11} \mathrm{erg} / \mathrm{rad}^{2}$ in diazomethane, compared to $0.086 \times 10^{-11} \mathrm{erg} / \mathrm{rad}^{2}$ in ketene and $0.23 \times 10^{-11} \mathrm{erg} / \mathrm{rad}^{2}$ in ethylene. The normality of the wagging force constant in ethylene and the size of the softenings in diazomethane and ketene have been previously rationalized. $F_{88}$, the out-of-plane bending force constant, is smaller in diazomethane $\left(0.53 \times 10^{-11}\right.$ $\left.\mathrm{erg} / \mathrm{rad}^{2}\right)$ than in ketene $\left(0.57 \times 10^{-11} \mathrm{erg} / \mathrm{rad}^{2}\right)$ and of lower value than the bending force constants of 16 electron triatomic molecules (greater than $0.65 \times 10^{-11}$ $\left.\mathrm{erg} / \mathrm{rad}^{2}\right)$. An unusual facet of the potential function is seen in the size of the interaction constant $F_{89}$. $A$ priori we should expect the magnitude of this interaction constant to increase roughly as the energy separation of the two modes in this class decreases. How- 
cver, the energy separation in $\mathrm{CH}_{2} \mathrm{~N}_{2}$ is $42 \mathrm{~cm}^{-1}$ and in $\mathrm{CH}_{2} \mathrm{CO}$ is $60 \mathrm{~cm}^{-1}$. The magnitudes of the interaction constants for these two systems are in the reverse direction one would expect: $0.0006( \pm 0.001) \times$ $10^{-11} \mathrm{erg} / \mathrm{rad}^{2}$ for diazomethane and $0.0527( \pm 0.001) \times$ $10^{-11} \mathrm{erg} / \mathrm{rad}^{2}$ for ketene. Although the validity of such interaction constants is in doubt because of the large anharmonicity in $\nu_{9}$, the observations are neatly explained in the pseudo-Jahn-Teller approach.

In the expansion of the Hamiltonian in $S_{i}$ and $S_{j}$ simultaneously, in second order the interaction constant becomes

$$
\begin{aligned}
F_{\boldsymbol{i} j}(k)= & \left\langle k\left|\mathfrak{H}_{\boldsymbol{i j}}\right| k\right\rangle \\
& -2 \sum_{\boldsymbol{s}}^{\prime}\left[\left\langle k\left|\mathfrak{H}_{\boldsymbol{i}}\right| s\right\rangle\left\langle s\left|\mathfrak{K}_{j}\right| k\right\rangle /\left(E_{k}{ }^{(0)}-E_{\boldsymbol{s}}{ }^{(0)}\right)\right],
\end{aligned}
$$

which, in the two-state approximation, becomes, for the electronic ground states,

$F_{i j}(0)=\left\langle 0\left|\mathfrak{F}_{i j}\right| 0\right\rangle-2\left(\left\langle 0\left|\mathfrak{K}_{i}\right| n\right\rangle\left\langle n\left|\mathfrak{K}_{j}\right| 0\right\rangle / \Delta \epsilon\right)$.

Comparison with Eqs. (1) and (2) gives us

$$
P_{i j}(n)^{2}=P_{i i}(n) P_{j j}(n) .
$$

Hence a symmetry interaction constant may also be softened if pseudo-Jahn-Teller effects are predicted in both the coupled modes. Since the pseudo-Jahn-Teller interaction is larger in diazomethane than in ketene, the softening of the interaction constant will be larger for the former than for ketene. In fact the interaction constant for diazomethane, $F_{89}$, is indistinguishable from zero.

In the computation of the softening of the interaction constant from Eq. (5), the larger softening in $F_{77}$ will dominate and the force constant $F_{67}$ is smaller (more negative) in ketene than in diazomethane. This explains the rather puzzling feature implied by the negative sign of this interaction constant. For such a value, the energy of the system is lowered in the cis configuration and raised in the trans, the opposite direction to that expected on considerations of steric hindrance. The argument presented here is probably a more generalized approach than Moore and Pimentel's suggestion that attractive forces exist between the hydrogen atoms and the skeletal electrons. ${ }^{22,23}$ The modes contained in the $A_{1}$ block require no further comment.

From the magnitude of the wagging force constant, these authors suggested that resonance form $\overline{\mathrm{C}} \mathrm{H}_{2}-\mathrm{N}^{+} \equiv \mathrm{N}$ contributed largely to the ground state of the molecule ${ }^{23}$ (analagously for ketene $\overline{\mathrm{C}} \mathrm{H}_{2}-\mathrm{C} \equiv \mathrm{O}^{+22}$ ). However, as we have seen, it is a structure of this type which is produced in the wagging mode and is stabilized by the transition charge density. Similarly, in the $\mathrm{CH}_{2}$ rocking motion, this structure is only stabilized when the hydrogen atoms are displaced from their equilibrium positions. Since one of the fundamental rules of resonance requires that all canonical forms must have similar values of molecular geometry parameters, the contribution of a "bent" canonical form to the actual molecular structure is excluded. The occurrence of a low-energy mode of this sort, produced by pseudo-Jahn-Teller interaction, is not therefore positive evidence for the large contribution of these two resonance forms to the structure of the electronic ground state. Similarly, a correlation of bond length and force constant will not be a viable argument when pseudo-Jahn-Teller interaction is considered, as is possibly indicated by the discrepancies in Decius' calculation via the relationship he gives. ${ }^{24}$

Another related system, treated by Moore and Rosengren, is hydrazoic acid $\mathrm{HN}_{3}{ }^{32}$ The main problems in this molecule, as far as force field work is concerned, are associated with the two linear modes of the skeleton and the $\mathrm{NH}$ wagging mode. Similar arguments may be applied to this system as have been invoked and discussed for diazomethane and ketene.

\section{Negative Anharmonicities in Diazomethane and Ketene}

Compared to the methyl radical, we are considerably restricted when it comes to choosing a basis set of electronic states to use in a calculation of the fourthorder terms in the potential-energy expansion since a definitive set of electronic states is not available. We will therefore initially consider a set containing the ground-state ${ }^{1} A_{1}$, two excited ${ }^{1} B_{2}$ states (which have been observed for diazomethane), one excited ${ }^{1} B_{1}$ state (only observed for diazomethane), and one ${ }^{1} A_{1}$ excited state (observed for both systems). As such these probably represent the lowest-lying states of the two systems.

For the $B_{2}$ block, the coefficient contribution to $S_{8,9^{4}}$ using the same parameterization as for carbon dioxide and ignoring for the moment the third term in Eq. (4) is

$$
-\frac{1}{4}\left(\beta^{2} / \epsilon\right)+4 \alpha^{2} \beta / \epsilon^{2}-4 \alpha^{4} / \epsilon^{3},
$$

where $\alpha=\left\langle{ }^{1} A_{1}\left|\mathfrak{H C}_{9}\right|{ }^{1} B_{2}\right\rangle ; \beta=\left\langle k\left|\mathfrak{F C}_{99}\right| k\right\rangle$; etc. This function is positive for all values of $\alpha^{2} / \epsilon>0.07 \beta$ up to the maximum permitted value of $\alpha^{2} / \epsilon=\frac{1}{4} \beta$ when the force constant in the mode becomes zero. For $\nu_{9}$ the softening is much larger in diazomethane than in ketene. Since this corresponds to a larger value of $\alpha^{2} / \epsilon$, the negative anharmonicities should be more pronounced in diazomethane than in the latter. This is indeed the case as can be seen from Table II. The inclusion of the third term in Eq. (4) should not have a very large effect as we have previously argued.

Negative anharmonicities are also observed in $\nu_{6}$ in ketene, the corresponding overtone band being unobserved in diazomethane. The situation here is analagous to that for $\nu_{9}$, except that only one excited perturbing state exists in our basis set. We should expect on the rational of the pseudo-Jahn-Teller effect that 
TABLE II. Anharmonicities in diazomethane and ketene."

\begin{tabular}{|c|c|c|c|c|c|c|}
\hline & \multicolumn{3}{|c|}{$\left(\mathrm{cm}^{-1}\right)$} & \multicolumn{3}{|c|}{$\left(\mathrm{cm}^{-1}\right)$} \\
\hline & $\mathrm{CH}_{2} \mathrm{~N}_{2}$ & $\mathrm{CHDN}_{2}$ & $\mathrm{CD}_{2} \mathrm{~N}_{2}$ & $\mathrm{CH}_{2} \mathrm{CO}$ & $\mathrm{CHDCO}$ & $\mathrm{CD}_{2} \mathrm{CO}$ \\
\hline$\nu_{9}$ & 409 & 368 & 318 & $528^{b}$ & 496.2 & 418.5 \\
\hline$\left(2 v_{9}\right)$ & 852 & 768 & 664 & $1062^{b, o}$ & $1003^{\mathrm{e}}$ & $870^{\mathrm{c}}$ \\
\hline$\Delta$ & +40 & +32 & +28 & +6 & +11 & +33 \\
\hline$\nu_{6}$ & $\ldots$ & $\ldots$ & $\cdots$ & 979 & $\ldots$ & $\ldots$ \\
\hline$\left(2 \nu_{6}\right)$ & ... & ... & $\ldots$ & 1979 & ... & ... \\
\hline$\Delta$ & $\cdots$ & $\cdots$ & $\begin{array}{c}\cdots \\
\Delta=\left(2 \nu_{6, \theta}\right)-2 \nu_{6,8}\end{array}$ & 26 & & \\
\hline
\end{tabular}

Gas-phase values, from Refs. 22 and 23.

b From Ref. 33. c $\left(2 \nu^{9}\right)$ in Fermi resonance with $\nu_{4}{ }^{33}$

contribution to the vibrational potential function up to fourth order in the displacement for a series of molecules. The qualitative treatment, involving no explicit evaluation of matrix elements, leads us to the conclusion that where a significant second-order softening (pseudo-Jahn-Teller) occurs we may expect to find unusual values of the third- and fourth-order terms in the potential expansion. In all the cases examined the electronic contribution to the fourth-order terms contained $\alpha^{2}$, the factor responsible for the second-order softening.

The present paper is thus able to rationalize several features of the vibrational problem which previous authors found puzzling, namely the negative anharmonicities in the methyl radical, diazomethane and ketene, the unusual force constants and interaction constants in the latter two systems, and the breakdown of the normal mode concept. It has presented an explanation of the negative interaction constants observed in some excited states of linear molecules of the $\mathrm{CO}_{2}$ type, and via the higher-order treatment has indicated that electron relaxation effects may well be important in determining the structure of the vibrational potential function in such linear molecules. In general it has tied together otherwise unrelated aspects of the theory of molecular vibrations, in those special situations where unusually low diagonal force constants are observed due to the pseudo-Jahn-Teller effect.

\section{ACKNOWLEDGMENT}

Sincere thanks are due to Dr. J. H. Current for his encouragement of this work and for several useful discussions.

* Power Foundation Fellow, Magdalene College, Cambridge, England.

1 R. F. W. Bader, Mol. Phys. 3, 137 (1960).

2 L. Salem, Chem. Phys, Letters 3, 99 (1969).

${ }^{3} \mathrm{G}$. Herzberg, Molecular Spectra and Molecular Structure. III

(D. Van Nostrand Co., Inc., Princeton, N.J. 1966), p. 345.

${ }^{4}$ D. E. Milligan, M. E. Jacox, and A. M. Bass, J. Chem. Phys. 43,3149 (1965). second-, third-, and fourth-order perturbation expansions to the determination of the electronic relaxation 
${ }^{5}$ J. W. C. Johns, Can. J. Phys. 39, 1738 (1961).

${ }^{6}$ J. W. C. Johns, Can. J. Phys. 42, 1004 (1964).

${ }^{7}$ I. C. Hisatsune and N. H. Suarez, Inorg. Chem. 3, 168 (1964).

${ }^{8}$ L. Gausset, G. Herzberg, A. Lagerquist, and B. Rosen, Astrophys. J. 145, 45 (1965).

${ }^{9}$ R. F. W. Bader, Can. J. Chem. 40, 1164 (1962).

${ }_{10}$ M. E. Jacox, D. E. Milligan, N. G. Moll, and W. E. Thompson, J. Chem. Phys. 43, 3734 (1965).

${ }_{11}$ D. E. Milligan and M. E. Jacox, J. Chem. Phys. 47, 5157 (1967). R. N. Dixon, Phil. Trans. Roy. Soc. (London) A252, $165(1960)$.

12 D. E. Milligan and M. E. Jacox, J. Chem. Phys. 44, 2850 (1966).

${ }^{13}$ D. E. Milligan and M. E. Jacox, J. Chem. Phys. 46, 703 (1967).

${ }_{14}$ A. M. Bass and D. E. Mann, J. Chem. Phys. 36, 3501 (1962).

${ }^{15}$ D. M. Dennison, Rev. Mod. Phys. 12, 175 (1940).

${ }^{16}$ M. E. Jacox and D. E. Milligan, J. Chem. Phys. 47, 5146 (1967).

${ }^{17}$ L. Andrews and G. C. Pimentel, J. Chem. Phys. 47, 3637 (1967)

${ }^{18}$ G. Herzberg, Proc. Roy. Soc. (London) A262, 291 (1961).

${ }^{19}$ R. W. Fessenden and R. H. Schuler, J. Chem. Phys. 39, 2147 (1963).
${ }^{20}$ A. D. Walsh, J. Chem. Soc. 1953, 2266

21 A. D. Walsh and P. A. Warsop, Trans. Faraday Soc. 57, 345 (1961)

${ }^{22}$ C. B. Moore and G. C. Pimentel, J. Chem. Phys. 40, 329, 342 (1964).

${ }^{23}$ C. B. Moore and G. C. Pimentel, J. Chem. Phys. 38, 2816 (1963).

${ }_{24}$ J. C. Decius, J. Chem. Phys. 45, 1069 (1966)

25 J. K. Burdett, J. Chem. Phys. (to be published)

${ }^{26}$ We use the convention of Moore and Pimentel in labeling the $B$ modes. This is not the convention suggested in Mulliken's report [J. Chem. Phys. 23, 1997 (1955)] and used by Merer. ${ }^{27}$

${ }_{27}$ A. J. Merer, Can. J. Phys. 42, 1242 (1964).

${ }^{28}$ R. G. W. Norrish, H. G. Crone, and O. D. Saltmarsh, J. Chem. Soc. 1933, 1533 .

${ }_{29}$ P. G. Wilkinson and R. S. Mulliken, J. Chem. Phys. 23, 1895 (1955).

${ }^{30}$ See Ref. 23

${ }^{31}$ S. M. Blinder, J. Chem. Phys. 35, 974 (1961) and references therein.

${ }^{32}$ C. B. Moore and K. Rosengren, J. Chem. Phys. 44, 4108 (1966).

${ }^{33}$ W. H. Fletcher and W. F. Arendale, J. Chem. Phys. 19, 1431 (1951).

THE JOURNAL OF CHEMICAL PHYSICS

VOL UME 52 , NUMBER 6

15 M A R C H 1970

\title{
Dissociation Energies, Enthalpies of Formation, Ionization Potentials, and Dipole Moments of NS and NS+*
}

\author{
P. A. G. O'HARE \\ Chemical Engineering Division, Argonne National Laboratory, Argonne, Illinois 60439
}

(Received 22 October 1969)

\begin{abstract}
A dissociation energy of $4.8 \pm 0.25 \mathrm{eV}$ at $0^{\circ} \mathrm{K}$ has been deduced for $\mathrm{NS}\left({ }^{2} \Pi\right)$ from spectroscopic data in the literature; the corresponding value for $\Delta H f_{0}{ }^{\circ}(\mathrm{NS})$ is $2.91 \pm 0.26 \mathrm{eV}$. Recent experimental results for $\Delta H f^{\circ}$ (NSF) from this Laboratory indicate $\Delta H f_{0}^{\circ}\left(\mathrm{NS}^{+},{ }^{1} \Sigma^{+}\right)=12.76 \pm 0.10 \mathrm{eV}$, from which the ionization potential of NS, $9.85 \pm 0.28 \mathrm{eV}$, is obtained. Hartree-Fock-Roothaan $a b$ initio calculations for NS and $\mathrm{NS}^{+}$yielded, respectively, first ionization potentials of 9.75 and $24.3 \mathrm{eV}$, dipole moments of 1.732 and $3.893 \mathrm{D}$, and, for $D_{0}{ }^{\circ}(\mathrm{NS})$, an approximate value of $5.2 \mathrm{eV}$.
\end{abstract}

\section{INTRODUCTION}

Nitrogen sulfide, NS, an unstable radical, is formed as an intermediate product by the reaction of active nitrogen with sulfur vapor ${ }^{1}$ or with sulfur chloride, ${ }^{2}$ or by passage of a high-frequency electrical discharge through a dilute mixture of sulfur hexafluoride in nitrogen. ${ }^{3}$ NS has been known for some time, ${ }^{4}$ and has been the subject of a number of spectroscopic investigations. $^{2-7}$ Apparently, no thermochemical properties have been determined due, undoubtedly, to the transient nature of the molecule. In the present paper, some thermochemical data for NS and also for $\mathrm{NS}^{+}$are deduced; the latter ion was first observed by Dressler ${ }^{8}$ in the course of a spectroscopic study.

We have recently reported ${ }^{9}$ the standard enthalpies of formation at $298.15^{\circ} \mathrm{K}, \Delta H f_{298^{\circ}}$, of thiazyl trifluoride $\left(\mathrm{NSF}_{3}\right)$ and thiazyl monofluoride (NSF). From these data and the electron-impact appearance potentials reported by Glemser et al., wo we have obtained $\Delta H f_{0}{ }^{\circ}\left(\mathrm{NS}^{+}\right)$. The latter result, combined with a value for $\Delta H f_{0}^{\circ}(\mathrm{NS})$ based on spectroscopic data, yielded the ionization potential of NS in the ground $\left({ }^{2} \Pi\right)$ state.

$A b$ initio calculations for $\mathrm{NS}$ and $\mathrm{NS}^{+}$, based on the Hartree-Fock-Roothaan approach, gave ionization potentials and dissociation energies in good agreement with the results derived from the experimental data.

\section{RESULTS FROM EXPERIMENTAL MEASUREMENTS}

\section{A. Dissociation Energy of NS}

A number of investigators ${ }^{2-8}$ have examined the emission spectrum of NS in detail. As a result, $\omega_{e}$ and $\omega_{e} x_{e}$ for the ground $\left({ }^{2} \Pi\right)$ state are quite well established at $1219 \pm 1$ and $7.5 \pm 0.2 \mathrm{~cm}^{-1}$, respectively. A linear Birge-Sponer extrapolation of the spectroscopic data yields $6.1 \mathrm{eV}$ for the dissociation energy, $D_{L B X}{ }^{\circ}$; this method, however, frequently gives erroneous results. ${ }^{11,12}$ Hildenbrand and Murad ${ }^{13}$ have indicated that dissociation energies obtained via the linear Birge-Sponer ex- 